\title{
Narrative Interfaces: Temporality and spatiality in art museums' online resources
}

\author{
Maribel Hidalgo Urbaneja \\ University of Glasgow \\ Glasgow G12 8QH, Scotland \\ m.hidalgo-urbaneja.1@research.gla.ac.uk
}

\begin{abstract}
Art museums use a variety of online resources to tell the stories of their artworks and collections. Online publications, online exhibitions, and other interactive resources have interfaces with distinctive temporal and spatial qualities that determine the way narratives are presented and users interact with them. Temporality and spatiality are related to usability of interfaces. This paper draws upon concepts from narratology and digital narratology and uses empirical data from a specialised audience of art history and visual arts scholars to discuss the significance of time and space in online resources' interfaces. The research interrogates the effectiveness the design and function of paratexts that help users to navigate online resources. Research results invite to rethink the relation between the visual and the verbal in online resources. The spatial disposition of illustrations, understood as paratextual elements, determines the mediality of a narrative. Lastly, it will be discussed how linearity and nonlinearity influences the interactions the user has with the resources. A linear continuous vertical interface requires more time from the user to be read and therefore is better suited for close reading than information seeking.
\end{abstract}

Narratology. Museum studies. Online resources. Interface. User experience.

\section{TIME AND SPACE IN NARRATIVES AND DIGITAL MUSEOLOGY}

Art museums use online resources to tell the stories of their artworks and collections. As well as these stories happen in historical time and space, their telling in online resources evolves over time and takes place in a space determined by the interface design. Narratology, the theory that studies narratives, distinguishes between temporal and spatial classes, that either belong to the story or the telling aspect of narratives. According to narratology the "time of narrating" (Genette 1972, pp.215-227) or "discourse time" (Chatman 1978, p.62) is the time of the narrating act, for example, the duration of an oral narration or the time spent in reading a narrative. In digital narratology, authors add another concept, the notion of "user time" (Koskimaa 2010, p.136), which concerns the time the user spends reading or engaging with the work. Regarding the spatial domain, narratology theorists enunciate the concept of "discourse space", understood as the "focus of spatial attention", the area to which the attention of the user is directed (Chatman 1978, p.102). But also the notion of "spatial extension of the text" which "refers to the spatiality of the text as a material object and to the dimensionality of the interface to with the reader, spectator or user" (Ryan 2014a).

Temporality and spatiality are interconnected; the extension of the text and its particular disposition in space requires or imposes a certain "reading" time from users. For example, if a text is broken down in paragraphs or not, if the size of the fonts used is smaller or bigger, or if it is arranged in a consecutive order, all those spatial dimensions influence the time spent with a text. All these dimensions are here understood as "paratexts" which are "verbal or other productions", and "intrinsic parts of the text" (Brookey \& Gray 2017), whose function is "to make present, to ensure the text's presence in the world, its reception and consumption" (Genette 1987, p.1). To be more precise, in books paratexts are constituted by elements including the author's name, covers, titles, prefaces, illustrations, notes, typography, and paper. The museum can be also regarded as a paratext. Paintings' frames, the wall space, illumination, "do not touch" signs become "parapaintings" (Cronin 2014). Finally, the notion of paratext expands to digital media; hence "interfaces, instructions, menus, statements, reviews, blog posts, and documentation belong to the new generation of paratexts" (Strehovec 2014, 
p.47). The interface is indeed one of the main paratexts in digital media. "Interface is part and parcel with paratext. It is one of the gateways we enter through to get things. In the digital era, you have all sorts of modifications of these gateways that we go through, and they are often like organizing systems" Jonathan Gray states (Brookey \& Gray 2017). This idea is shared by Johanna Drucker's who makes a similar affirmation, according to her the interface is "a space of affordances and possibilities (...) a set of conditions, structured relations" that enable readings and user interaction" (Drucker 2013).

The concepts of "discourse time", "discourse space", "spatial extension of the text", and "paratext" are implicit in recent discussions on digital storytelling and narratives in museums. Museums are concerned with the effectiveness of the interfaces of their websites. Different text lengths, buttons, menus, the prevalence of images over text or vice versa, among other paratextual elements, posit challenges to the reading experience in web pages. Museum blogs argue that interfaces should support layered storytelling that integrates text, images, and other multimedia contents, and "make the 'read more' or 'related stories' content more inviting" without "breaking the reading experience" (Grant 2014). The blog post mentions best practices and examples drawn from other sectors, namely journalism, as possible solutions. This presupposes that museums' online resources are not as optimised as they should be for digital storytelling, but is there an evidence of what paratextual elements do not function as they should?

Because the digital medium commonly involves fast paced interactions of the user with the text, museums are concerned with user time. The short duration of digital "visits" would imply a lack of engagement of users who "snack" content and go from page to page clicking on hyperlinks (Birchall \& Faherty 2016). To weave "moments of contemplation" into the narrative flow (Kraemer 2007), similar to the ones visitors experience in a physical museum, may stretch online visits. This is achieved through modifications of discourse space and the spatial extension of the contents. Some museums' online resources use vertical linear interfaces and parallax effects to "restore a sense of the scale and pace of museum experiences" (Birchall and Faherty 2016). In this way, the narrative would invite the audience to slow down the online visit and read the text word by word, step by step (Birchall \& Faherty 2016). Yet, some question the efficacy of the linear parallax based model and state that users tend to skim long pieces of content (Samis \& Svenonius 2015). The contradictory opinion suggests that more analysis and comparison between multi page and vertical linear websites is needed to understand the implications of different user times and interfaces design.

\section{METHODOLOGY}

The research that this paper presents is part of a PhD research focused on a definition of art museums' online resources as digital narratives. A methodological approach that applies narratology to empirical data is used to study the different components of narratives, from authorship and readership, to temporality and spatiality - the two notions this paper discusses.

In order to collect the research data, six online resources from major museums were selected from a survey of online publications, online exhibitions, exhibition websites, and other interactive online resources. The selection includes resources from the US (Metropolitan Museum of Art 2013, Abbaspour et al. 2014, National Gallery of Art 2014), Spain (Museo Nacional del Prado 2016, Museo Nacional Centro de Arte Reina Sofía 2016), and the UK (Lillie 2014). The results here presented are based on empirical data collected from twenty individuals as they perform think-aloud protocol while visiting two different online resources from the six selected. These individuals represent a specialised audience of art history and visual arts scholars at different career stages, from doctoral candidates to retired professors. The perspectives of participants reflect preferences, needs, and behaviours related to their teaching and research activities.

\section{NAVIGATION AND ORIENTATION: PARATEXTS}

There exists a connection between the navigation and the completeness of an online resource, which converses with $\mathrm{HCl}$ approaches that use narratives as a method to assess usability (Brejcha 2015). As Brejcha explains, this approach understands the argument presented in a multi-page website as a global narrative with which a user interacts: "The global narration is supported by the navigation and interaction component running through a sequence of screens. These elements do not perceptually appear in some static form, but are enacted through the user's activities with the computer" (Brejcha 2015, pp.36-37). This method can help evaluate the flow of navigation of an online resource and whether users are able to reach all its parts or these remain hidden to them. In navigating an online resource, paratextual elements are the "elements" users enact. "[N]ew media paratexts are also important in the form of menus and instructions that facilitate orientation and progress 
in traversing the e-text scape, which means that they are also essential for navigating and controlling the literary text" (Strehovec 2014). This quotation, although relative to literary works, summarises the relevance of menus, buttons, banners, and other orienting paratexts in websites. These paratextual elements operate at the level of "discourse space" and spatially distributed in consonance with the text. The importance of these paratexts in art museums' online resources was revealed during the interaction of participants with four of the six online resources here studiedgiven that only these four online resources are multi-page, and the other two are single paged. Interestingly, results pertinent to these orientational paratexts emerged when participants struggled with navigation. In these instances participants expressed their intention to return to previous pages but could not perform the action, or were hesitant about what their next action would prompt.

Like a visitor in a museum who needs a map or to ask visitor service staff for hints, users of multipage complex websites can feel disoriented. This was evident in participants' interactions:

\section{Now I kind of I don't know where I am in the website and how I can get back to where I was before. (Participant 7 2016)}

Multi-page online resources present a "tree structure" (Ryan 2014b, p.240), in which a home page would be the entry point, the "roots" of the tree, and the "branches" are the pages that emerge from that root. This structure often invites users to continue visiting "branches" but also return to the "root". The online resources studied either presented a breadcrumbs menu or regular menus to facilitate navigation back to the home page, yet these elements were ignored by participants. Often participants used the back button of the browser to return to the previous visited page: "then, to go back to where I was (...) I use the button, the back button" (Participant 3 2016). In general this action provided the expected result, although sometimes participants were frustrated because they could not return to the previously page visited. This issue denotes a need to further improve overall navigation of online resources.

Menus, buttons, and hyperlinks are key elements used to advance navigation in online resources. If these elements are not positioned in the right place, are not noticeable, or confusing, users struggle to make progress. During think-aloud sessions participants noted when they were unsure about how to obtain further information, and therefore progress in the narrative: "I'm missing something here that gives a bit more information" (Participant 13 2016). In these cases, hints were given to them in order to continue navigation.
Participants were also hesitant about what kind of functions would be triggered when clicking on some hyperlinks or buttons. This is something that can prevent them from continuing a visit as Participant 11 explains:
I guess sometimes I can get frustrated pretty easily (...) if it's not clear what it's happening, where you go next, or something like that. I feel like I would stop looking at it, instead to try to spend a lot of time figuring out. Sometimes it's laid out in a confusing way I don't understand where it's the space of it, like where it's the next part. (Participant 11 2016)

Only very few participants provided specific reasons that explain such issues would emerge during navigation. For example Participant 18 Clarifies:

It makes you, with these sorts of small links, I think, less likely to click on them because (...) it's going to be more details or information, and not anything interactive like this. (Participant 18 2016)

This shows that paratextual elements, namely the size and design choice of buttons and text fonts, determine whether users reach all contents of the online resource, and therefore construct a complete narrative. Missing or discovering elements of a narrative changes users' perception of it. The design of a user experience approach that takes into account the interplay between paratexts and the flow and completeness of a narrative can help to improve the navigation of online resources.

\section{THE VISUAL AND THE VERBAL: ILLUSTRATIONS}

Illustrations are paratexts considered visual "productions" that either reinforce or accompany a text (Genette 1991). The notion of illustration can be further divided into "two basic ways of illustrating": the one in which the visual underlines the verbal level of the text, and the second in which the image becomes a "text per se" (Baetens 2003 p.187). In online resources that present narratives around objects, the tension between the visual and the verbal invite to reconsider whether images are illustrations that support the text, and therefore are paratexts, or if images stand by themselves. The illustrative function of images has much to do with their space and position in web pages. This schema also helps to examine mediality in online resources enriching a long-held debate on "images and text" (Engberg 2014) (Hocks and Kendrick 2003).

While some online resources from the six selected are more visual, others are substantially more verbal. Participants can notice this because the 
resource provides more space to one medium than the other. This is particularly manifest with online publications. Participants highlighted when they were in front of a publication because images of artworks were used as illustrations subordinated to the text:

The work is an illustration because (...) rather than driving force they are illustrations of an art historical text. (Participant 2 2016)

I'm drawn much more to the text than I am to the image. The image around seems to be secondary on these pages. (Participant 18 2016)

Although this is common in publications, participants ask for a more balanced relationship between image and text. They think images should be equally or more important than the text and this translates in terms of image resolution and spatial configuration. For example, one of them explains

If you don't have a good image associated to textual content, the value it's pretty small unfortunately. (Participant 16 2016.)

If a text is not supported by a high-resolution and prominent image, the verbal discourse is incomplete or ineffective. Another participant shares the same opinion:

I'm looking (...) at the cropped image, which is static, which is tiny, and there are no dimensions on the caption that they've got and it's (...) kind of undermining what they're trying to do. (Participant 2 2016.)

The same participant remarks that an illustrative approach to images puts the intrinsic qualities of the artwork "at distance".

Making images more relevant calls into question the illustrative model that serves the verbal component of online resources. Baetens schema and research results invite to treat most images of artworks as standalone elements in online resources. After all artworks themselves are texts, which can be eventually "read" and viewed without verbal aids. This can be done augmenting the quality and size of the artworks' images, which changes their spatial configuration, and designing interfaces that are more sympathetic with the visual. For authors of online resources, considering the images of artworks from an illustrative and paratextual perspective can help create more coherent online resources and enhancing the medial qualities of their discourses.

\section{LINEARITY AND NONLINEARITY: INTERFACES AND USER TIME}

Specific spatial features of interfaces invite to interaction at different paces. Three of the six studied online resources use the vertical parallax scrolling model. The three others feature a more compact design that does not require excessive scrolling or not scrolling at all. Vertical scrolling is considered an "effortless" form of interaction (Bostock 2014) and the data from participants confirms this idea:

I like its one page and you just have to scroll down and (...) don't have to click too much. All the information is on this one website and you can (...) scroll through the images so it's really easy. (Participant 19 2017)

The data from participants also reinforces some ideas introducing this paper, in particular that this form of interaction, slows down online visits (Birchall \& Faherty 2016) imposing a certain "discourse time". A participant explains:

The information was presented in such a way to slow down enough while also scrolling through the art. (Participant 18 2016)

Overall, linear interfaces suit the slow paced and detail oriented interactions required by close reading.

However, because the participants of this research are scholars and their visits to art museums' online resources are primarily motivated by research and teaching purposes, obtaining information quickly is important. Their "user time" may be in conflict with the "discourse time" of the resource. Participants recognised the importance of time in their online visits, so as participant number three explains: "I'm very keen to get information quickly" (Participant 3 2016). Online resources present a clear advantage for them:

You can get the information very immediately, because otherwise you have to go the stacks and all these things." (Participant 20 2016)

The pressing need to get information rapidly makes vertical scrolling down interfaces not be the best suited for effective information search. Participant number twelve notices that this linear vertical model has:

A kind of structure where you don't get an overview of what you're looking at (...) if you do research or trying to find a specific work (...) and maybe want to go back find something you saw, it would be really difficult to do that I think. (Participant 12 2016)

The same participant compares the linear vertical model with a more compact and traditional interface, in which there is no necessity to scroll to see all contents, and points that in the second type of interface gets: 
This basic page and you can just look at it for a few minutes and then you can go further. (Participant 12 2016.)

Interestingly, this argument is not completely new and not so recent usability manuals share the same opinion. Nielsen (2000, p.115) argues that vertical linear interfaces are not good for information search.

The negative connotations of rapid navigation on the Web that emerge from the works cited at the beginning of this paper contrast with the behaviours displayed by the participants of this research. As said, they praise immediacy when they browse online resources, but when they find relevant information may spend time with it reading it closer. They scan the web pages to assess whether a page contains information that is relevant to them to decide whether to spend more time with it. As Participant 11 explains:

You see just a little bit of information and you can go further. (Participant 11 2016)

Participants fixed their attention on paratextual elements, titles, menus, illustrations, and bibliography, instead of reading long blocks of text or essays. For example, as soon as opening a new page, a participant scrolled down to the bottom of it and verbalised that was looking at the bibliography before reading the essay as a way to understand whether the essay is worth attention:

I'm checking the sources, I'm just looking what they're citing. (Participant 19 2017)

Understanding the extension of the site and its scope is key to decide where to go next and what details to pay attention to. As Participant 16 explains, "I kind of like move my cursor around to see what is there" (Participant 16 2016) and later in the session points out to the fact that in the visited resource:

They have a really long bibliography, so let's look at what has been done recently... so maybe this is classic thing that art historians get caught off in the content and stop investigating the website. (Participant 16 2016)

This example conveys that scanning, skimming, or "snaking" content, in sum, non linear navigation on a website can lead also to engaged interactions. Online navigation can be a combination of both quick and slow interactions. This contradicts the opinion of some previously cited authors (Birchall \& Faherty 2016) (Samis \& Svenious 2015) and highlights a challenge museums face to harness the design of interfaces that allow close reading and search.

\section{CONCLUSION: NARRATIVES AND USABILITY}

Temporality and spatiality are more than the time and space in which a story takes happens, the temporal and spatial dimensions that interfaces introduce to narratives determine their "reading", the interaction of the user with it. This research shows how the introduction of narratology concepts in user experience research can provide valuable insights to improve the interfaces of art museums' online resources. Looking at online resources through the lenses of narratology concepts on time and space:

- Helps to evaluate whether navigation across multi-page websites is consistent and cohesive.

- Helps to understand if visuality reinforces the narrative and fulfils its role.

- Provides evidence about the close connection between the spatial dimensions of an interface and temporality.

It is also necessary to mention that even though this research focuses on a specific segment of the audience, a scholarly audience, theory and methods can be used with other types of users. General audiences are also affected by the same issues. This should invite museum professionals to apply such methods in the design and evaluation of online resources.

\section{REFERENCES}

Abbaspour, M., Daffner, L. A. and Morris Hamburg M. (2014) Object:Photo. Modern Photographs: The Thomas Walther Collection 1909-1949 at The Museum of Modern Art.

https://www.moma.org/interactives/objectphoto/\#ho me (retrieved 12 March 2019).

Baetens, J. (2003) Illustrations, images, and antiillustrations. In Hocks, M. E. and Kendrick, M. R. (eds). Eloquent Images: Word and Image in the Age of New Media. The MIT Press, Cambridge, MA.

Birchall, D. and Faherty A. (2016) Big and slow: Adventures in digital storytelling. MW2016: Museums and the Web 2016.

http://mw2016.museumsandtheweb.com/paper/bigand-slow-adventures-in-digital-storytelling/ (retrieved 12 March 2019).

Bostock, M. (2014) How To Scroll. Mike Bostock. [personal site]. https://bost.ocks.org/mike/scroll/ (retrieved 12 March 2019).

Brejcha, J. (2015) Cross-Cultural Human-Computer Interaction and User Experience Design. CRC Press, Boca Raton, FL. 
Brookey, R. and Gray. J. (2017) Not merely para: continuing steps in paratextual research. Critical Studies in Media Communication 34 (5), pp.101110. doi:10.1080/15295036.2017.1312472

Chatman, S. (1978) Story and Discourse: Narrative Structure in Fiction and Film. Cornell UP, Ithaca.

Cronin, B. (2014) Foreword. In Desrochers, N. and Apollon, D. (eds). Examining Paratextual Theory and Its Applications in Digital Culture. IGI Global, Pennsylvania.

Drucker, J. (2014) Graphesis. Visual Forms of Knowledge Production. Harvard University Press, Cambridge, MA.

Drucker, J. (2013) Performative Materiality and Theoretical Approaches to Interface. Digital Humanities Quarterly 7(1).

http://www.digitalhumanities.org/dhq/vol/7/1/00014 3/000143.html (retrieved 12 March 2019).

Engberg, M. (2014) Word-Image. In Emerson, L., Ryan, M., and Robertson, B. J. (eds). The Johns Hopkins Guide to Digital Media. Johns Hopkins University Press, Baltimore.

Genette, G. ([1972] 1980) Narrative Discourse: An Essay in Method. Cornell UP, Ithaca.

Genette, G. (1991) Introduction to the Paratext. New Literary History 22 (2), pp.261-72. doi:10.2307/469037.

Grant, F. (2014) Approaches to Online Storytelling. SFMOMA Blog.

https://www.sfmoma.org/read/approaches-onlinestorytelling/ (retrieved 12 March 2019).

Hocks, M. E. and Kendrick M. R. (2003) Eloquent Images: Word and Image in the Age of New Media. MIT Press, Cambridge, MA.

Koskimaa, R. (2010) Approaches to Digital Literature: Temporal Dynamics and Cyborg Authors. In Simanowski, R., Schåfer, J. and Gendolla, P. (eds). Reading Moving Letters: Digital Literature in Research and Teaching.Transcript, Bielefeld, Germany. pp.129-143.

Kraemer, H. (2007) Simplicity, Slowness and Good Old Stories as Strategies and Perspectives of Design in Hypermedia and Media. International Cultural Heritage Informatics Meeting (ICHIM07): Proceedings.

http://www.archimuse.com/ichim07/papers/kraemer Ikraemer.html (retrieved 12 March 2019).

Lillie, A. (2014) Building the Picture. Architecture in Italian Renaissance Painting.

https://www.nationalgallery.org.uk/paintings/researc h/exhibition-catalogues/building-the-picture (retrieved 12 March 2019).

Metropolitan Museum of Art (2013) 82nd and Fifth. https://82nd-and-fifth.metmuseum.org/ (retrieved 12 March 2019).

Museo Nacional del Prado (2016) Bosh. A story in pictures. https://www.museodelprado.es/en/whats- on/exhibition/bosch-the-5th-centenaryexhibition/f049c260-888a-4ff1-8911-b320f587324a (retrieved 12 March 2019).

Museo Nacional Centro de Arte Reina Sofía (2016) Featured artworks.

http://www.museoreinasofia.es/en/featuredartworks (retrieved 12 March 2019).

National Gallery of Art (2014) Online Editions. https://www.nga.gov/research/online-editions.html (retrieved 12 March 2019).

Nielsen, J. (2000) Designing web usability: The practice of simplicity. New Riders Publishing.

Participant 2 (2016) Think aloud session led by author. November 17th, 2016.

Participant 3 (2016) Think aloud session led by author. October 10th, 2016.

Participant 7 (2016) Think aloud session led by author. July 16th, 2016.

Participant 11 (2016) Think aloud session led by author. July 18th, 2016

Participant 12 (2016) Think aloud session led by author. October 28th, 2016.

Participant 13 Think aloud session led by author. June 12th, 2016.

Participant 16 (2016) Think aloud session led by author. July 14th, 2016.

Participant 18 (2016) Think aloud session led by author. October 25th, 2016.

Participant 19 (2017) Think aloud session led by author. February 2nd, 2017.

Participant 20 (2016) Think aloud session led by author. July 12th, 2016

Ryan, M. (2014a) Space. In Hühn, P. et al. (eds). The living handbook of narratology. Hamburg University, Hamburg. http://www.Ihn.unihamburg.de/article/space (retrieved 12 March 2019).

Ryan, M. (2014b) Graph theory. In Emerson, L., Ryan, M., and Robertson, B. J. (eds). The Johns Hopkins Guide to Digital Media. Johns Hopkins University Press, Baltimore.

Samis, P. and Svenonius, T. (2015) The X, Y, and $Z$ of digital storytelling: Dramaturgy, directionality, and design. MW2015: Museums and the Web 2015.

http://mw2015.museumsandtheweb.com/paper/thex-y-z-of-digital-storytelling-dramaturgydirectionality-and-design/ (retrieved 12 March 2019).

Strehovec, J. (2014) E-Literary Text and New Media Paratexts. In Desrochers, N. and Apollon, D. (eds). Examining Paratextual Theory and Its Applications in Digital Culture. IGI Global, Pennsylvania. 